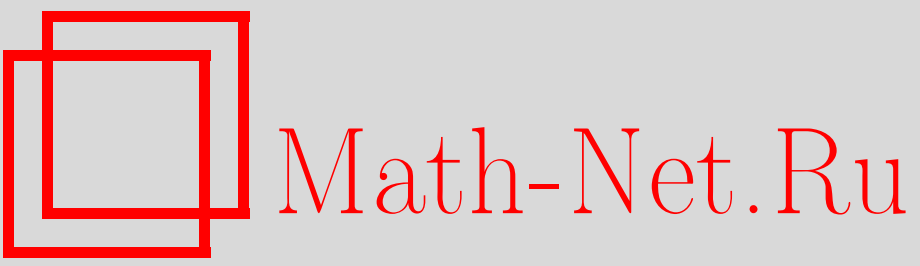

В. И. Солодовников, О примарных функциях, минимально близких к линейным, Матем. вопр. криптогр., 2011, том 2, выпуск 4, 97-108

DOI: https://doi.org/10.4213/mvk45

Использование Общероссийского математического портала Math-Net.Ru подразумевает, что вы прочитали и согласны с пользовательским соглашением

http://www.mathnet.ru/rus/agreement

Параметры загрузки:

IP : 54.224 .135 .184

26 апреля 2023 г., 02:53:22 


\author{
МАТЕМАТИЧЕСКИЕ ВОПРОСЫ КРИПТОГРАФИИ \\ 2011 T. 2 № 4 C. 97-108
}

УДК 519.719 .2

\title{
О примарных функциях, минимально близких к линейным
}

\author{
В. И. Солодовников \\ Академия криптографии Российской Федерации, Москва
}

Получено 23.VI.1010

Продолжается начатое автором ранее исследование вопросов близости функций из $(\mathbf{Z} /(p))^{n}$ в $(\mathbf{Z} /(p))^{m}$ (p- простое) к линейным функциям. Найдены новые критерии минимальной близости функции к линейным. Доказывается, что такая минимальность функции наследуется ее гомоморфными образами. В качестве обобщения утверждения, хорошо известного для булевых функций, доказывается, что для $p=2,3$ класс всех минимально близких к линейным функций совпадает с классом бент-функций (в случае их существования).

Ключевые слова: близость функций, абсолютно негомоморфные функции, минимальные функции, бент-функции

\section{On primary functions which are minimally close to linear functions}

\section{I. Solodovnikov}

Academy of Cryptography of Russian Federation, Moscow

\begin{abstract}
The investigation of aspects of closeness to linear functions for functions from $(\mathbf{Z} /(p))^{n}$ to $(\mathbf{Z} /(p))^{m}$ (p is prime number). New criteria of minimal closeness to linear functions are found. This property of a function is proved to be inherited for its homomorphic images. As a generalization of an analogous statement for Boolean functions it is proved that if $p=2$ or 3 then a class of functions which are minimally close to linear ones coincides with the class of bent-functions (if bent-functions do exist).
\end{abstract}

Key words: closeness of functions, absolutely nonhomomorphic functions, minimal functions, bent-functions

Citation: Mathematical Aspects of Cryptography, 2011, vol. 2, no. 4, pp. 97-108 (Russian). 
Нам потребуются следующие обозначения, терминология и результаты работы [5].

$X, Y$ - аддитивные конечные абелевы группы.

$Y^{X}$ - множество всех отображений $f: X \rightarrow Y$ из множества $X$ в множество $Y$ (термины «отображение» и «функция» считаем синонимами).

$\psi \varphi$ - композиция отображений, при которой $\varphi$ действует первым.

$\operatorname{Hom}(G, H)$ - множество всех гомоморфизмов группы $G$ в группу $H$.

$\boldsymbol{Z}$ - кольцо целых чисел, $\boldsymbol{Z} /(k)$ - кольцо вычетов по модулю $k$.

$P$ - равномерное вероятностное распределение на $X$, то есть

$$
P(A)=|A||X|^{-1}
$$

для любого $A \subseteq X$.

Функция $f: X \rightarrow Y$ называется сбалансированной, если мощности полных прообразов всех элементов $Y$ одинаковы.

В работе [5] близость двух произвольных функций $f_{1}, f_{2} \in Y^{X}$ измерялась корнем из дисперсии

$$
\left(|Y|^{-1} \sum_{y \in Y}\left(P\left(f_{1}-f_{2}=y\right)-|Y|^{-1}\right)^{2}\right)^{\frac{1}{2}} .
$$

В [6] была предложена другая нормировка этой величины, при которой ее максимальное значение становится равным 1:

$$
\left(|Y|(|Y|-1)^{-1} \sum_{y \in Y}\left(P\left(f_{1}-f_{2}=y\right)-|Y|^{-1}\right)^{2}\right)^{\frac{1}{2}} .
$$

Это позволило упростить многие формулы. С целью дальнейших упрощений здесь мы откажемся и от корня. А именно, близость $\boldsymbol{\delta}$ функций $f_{1}$, $f_{2} \in Y^{X}$ определим равенством

$$
\delta\left(f_{1} f_{2}\right)=|Y|(|Y|-1)^{-1} \sum_{y \in Y}\left(P\left(f_{1}-f_{2}=y\right)-|Y|^{-1}\right)^{2},
$$

а близость классов функций $K_{1}, K_{2} \subseteq Y^{X}-$ равенством

$$
\delta\left(K_{1}, K_{2}\right)=\max _{f_{1} \in K_{1}, f_{2} \in K_{2}} \delta\left(f_{1}, f_{2}\right),
$$

Близость $\delta$ обладает следующими свойствами:

$$
\begin{gathered}
0 \leq \delta\left(f_{1}, f_{2}\right) \leq 1, \\
\delta\left(f_{1}, f_{2}\right)=0 \Leftrightarrow f_{1}-f_{2}-\text { сбалансированная, } \\
\delta\left(f_{1}, f_{2}\right)=1 \Leftrightarrow f_{1}-f_{2}=\text { const, }
\end{gathered}
$$




$$
\begin{gathered}
\delta\left(f_{1}, f_{2}\right)=\delta\left(f_{2}, f_{1}\right), \\
\delta\left(y_{1}+g_{2} f_{1} g_{1}+f^{\prime}, y_{2}+g_{2} f_{2} g_{1}+f^{\prime}\right)=\delta\left(f_{1}, f_{2}\right)
\end{gathered}
$$

для любых $f_{1}, f_{2} \in Y^{X}$, где $g_{1}: X^{\prime} \rightarrow X-$ сбалансированная, $g_{2}: Y \rightarrow Y^{\prime}-$ изоморфизм, $f^{\prime}: X^{\prime} \rightarrow Y^{\prime}, y_{1}, y_{2} \in Y^{\prime}$.

Свойства (2), (3), (4) означают, что минимально близкие функции это функции, разность которых сбалансирована, а максимально близкие функции — это функции, разность которых есть константа.

Для любого $a \in X$ подстановку $x \mathrm{l} \rightarrow a+x$ множества $X$ будем обозначать через $a^{+}$. Все такие подстановки называют сдвигами группы $X$. Они образуют изоморфную группе $X$ группу подстановок, называемую группой сдвигов (или группой Кэли) группы $X$ (в теории представлений групп такое представление группы $X$ называют регулярным).

Гомоморфизмы $h \in \operatorname{Hom}(X, Y)$ по определению обладают следующим характерным свойством: $h a^{+}-h=$ const для всех $a \in X \backslash\{0\}$. Поэтому естественным (в силу свойств (2), (3), (4)) является следующее определение, обобщающее определение «совершенной нелинейности» в [9].

Функиия $f: X \rightarrow Y$ называется абсолютно негомоморфной, если для любого $а \in X \backslash\{0\}$ функиия $f a^{+}-f$ является сбалансированной, то есть

$$
\delta\left(f a^{+}, f\right)=0 .
$$

Множество всех абсолютно негомоморфных функиий из $Y^{X}$ обозначается через $\boldsymbol{B}(\boldsymbol{X}, \boldsymbol{Y})$.

К понятию абсолютной негомоморфности можно подойти не только с алгебраической, но и с криптографической стороны. А именно, пусть $G-$ некоторая группа подстановок множества $X, f G=\{f g \mid g \in G\}-$ класс функций, порождённых функцией $f \in Y^{X}$ и группой $G$. Пусть в некоторой криптосхеме имеется узел, реализующий функции из $f G$, где элементы группы $G$ являются ключами. Тогда величины $\delta\left(f g_{1}, f g_{2}\right), g_{1}, g_{2} \in G$, характеризуют степень изменения этого узла ключами из $G$ : чем ближе эти величины к 0 , тем сильнее подстановки из $G$ изменяют функцию $f$. Наоборот, если величина $\delta\left(f g_{1}, f g_{2}\right)$ близка к 1 , то ключи $g_{1}$ и $g_{2}$ называют близкими. Поскольку, в силу (6), $\delta\left(f g_{1}, f g_{2}\right)=\delta\left(f g_{1} g_{2}^{-1}, f\right)$, то достаточно рассматривать только величину $\delta(f g, f), g \in G$. В частности, когда $G$ - группа сдвигов группы $X$, получаем, что абсолютно негомоморфные функции - это функции, которые максимально изменяются сдвигами, то есть соответствующие узлы не имеют различных близких ключей-сдвигов. $f \in Y^{X}$

В работе [5] доказано, что если группа $G$ транзитивна, то для любой

$$
\delta(f, 0)=|Y|(|G|(|Y|-1))^{-1} \sum_{g \in G}\left(P(f g=f)-|Y|^{-1}\right)
$$


и, в частности, для любого $h \in \operatorname{Hom}(X, Y)$

$$
\delta(f, h)=|Y|(|X|(|Y|-1))^{-1} \sum_{a \in X}\left(P\left(f a^{+} f=h(a)\right)-|Y|^{-1}\right) .
$$

Приведём некоторые свойства абсолютно негомоморфных функций: если $f \in B(X, Y), h \in \operatorname{Hom}(X, Y), g_{1}: X^{\prime} \rightarrow X-$ изоморфизм, $g_{2}: Y \rightarrow Y^{\prime}-$ эпиморфизм (т.е. сюръективный гомоморфизм групn), $c \in X^{\prime}, \quad b \in Y^{\prime}$, $h^{\prime} \in \operatorname{Hom}\left(X^{\prime}, Y^{\prime}\right), m o$

$$
\begin{gathered}
\delta(f, h)=|X|^{-1}, \\
b+h^{\prime}+g_{2} f g_{1} c^{+} \in B\left(X^{\prime}, Y^{\prime}\right) .
\end{gathered}
$$

Из формулы (9) следует, что абсолютно негомоморфные функции одинаково близки к любому гомоморфизму, а также они не являются сбалансированными при $|Y|>1$, поскольку сбалансированность равносильна условию

$$
\delta(f, 0)=0 .
$$

В частности, абсолютно негомоморфные функции не могут быть биективными.

В соответствии с теоретико-автоматной терминологией и с учетом групповой структуры алфавитов, пару $(\alpha, \beta)$ гомоморфизмов $\alpha \in \operatorname{Hom}(X, X)$ и $\beta \in \operatorname{Hom}(Y, Y)$ назовем гомоморфизмом функиии $f: X \rightarrow Y$ в функиию $f^{\prime}: X^{\prime} \rightarrow Y^{\prime}$, если $\beta f=f^{\prime} \alpha$. Если $\alpha$ и $\beta$ - сюръекции, то гомоморфизм $(\alpha, \beta)$ назовем эпиморфизмом, а функцию $f^{\prime}-$ гомоморфным образом функиии $f$. В этом случае для любых $a \in X$ и $y^{\prime} \in Y^{\prime}$

$$
P^{\prime}\left(f^{\prime}(\alpha(a))^{+}-f^{\prime}=y^{\prime}\right)-\left|Y^{\prime}\right|^{-1}=\sum_{y \in \beta^{-1}\left(y^{\prime}\right)}\left(P\left(f a^{+}-f=y\right)-|Y|^{-1}\right)
$$

(где $P^{\prime}\left(A^{\prime}\right)=\left|A^{\prime}\right|\left|X^{\prime}\right|^{-1}$ для любого $\left.A^{\prime} \subseteq X^{\prime}\right)$. Легко проверяется справедливость следующего утверждения.

Теорема 1. Если $(\alpha, \beta)$ - эпиморфизм функциии $f \in B(X, Y)$ в функциюю $f^{\prime}: X^{\prime} \rightarrow Y^{\prime}$ и $\left|Y^{\prime}\right|>1$, по $\alpha-$-изоморфизм и $f^{\prime} \in B\left(X^{\prime}, Y^{\prime}\right)$.

Минимальная близость функций из $Y^{X}$ к гомоморфизмам определяется равенством

$$
\delta_{0}(X, Y)=\min _{f \in Y^{X}} \delta(f, \operatorname{Hom}(X, Y)) .
$$


Минимальными называются функции, минимально близкие к гомоморфизмам, то есть функции $f \in Y^{X}$, для которых

$$
\delta(f, \operatorname{Hom}(X, Y))=\delta_{0}(X, Y) .
$$

Множество всех минимальных функций из $Y^{X}$ обозначим через $\boldsymbol{M}(\boldsymbol{X}, \boldsymbol{Y})$.

Заметим, что минимальные функции существуют всегда, в отличие от абсолютно негомоморфных функций.

Хорошо известно, что любая конечная абелева группа изоморфна прямому произведению аддитивных групп колец вычетов. Поэтому без ограничения общности (в силу формул (6) и (10)) далее считаем, что

$$
X=\prod_{i=1}^{n} \boldsymbol{Z} /\left(k_{i}\right), \quad Y=\prod_{j=1}^{m} \boldsymbol{Z} /\left(t_{j}\right),
$$

где $n, m-$ произвольные натуральные числа, $k_{1}, \ldots, k_{n}, t_{1}, \ldots, t_{m}-$ произвольные натуральные числа, большие 1 .

В работе [5] для функций из $Y^{X}$ введено понятие бент-функции как функции, обладающей следующим свойством: в разложении композиции ее илюбого неединичного неприводимого (комплекснозначного) характера группы Y по неприводимым характерам группы Х модули всех коэффиииентов (они называются коэффициентами Фурье) одинаковы. Это определение обобщает определения работы [2] (для булевых функций, когда $m=1$, $k_{1}=\ldots=k_{n}=t_{1}=2$ ), работы [3] (когда $k_{1}=\ldots=k_{n}=t_{1}=\ldots=t_{m}=p$ - простое число, $m$ делит $n$ ) и может быть сведено к определению работы [4], где бентфункции определяются как комплекснозначные функции на конечной абелевой группе с единичными модулями всех значений и условием равенства модулей всех коэффициентов Фурье. Для случая $m=1, k_{1}=\ldots=k_{n}=t_{1} \geq 3$ определение из [5] сужает определение бент-функции в [8] (где участвует только один характер группы $Y$ ), но в [8] доказано, что при простом $t_{1}$ эти определения равносильны. В книге [10] приводится обстоятельный обзор бент-тематики.

В [5] доказано:

множество всех бент-функций из $Y^{X}$ совпадает с $B(X, Y)$, что распространяет результаты [2], [3], [8] на произвольные конечные абелевы группы $X$ и $Y$.

Для любого $h \in \operatorname{Hom}(X, Y)$ матрииа гомоморфизма $h$

$$
A_{h}=\left(a_{i, j}+\left(t_{j}\right)\right)_{n \times m}
$$


определяется равенствами

$$
\begin{gathered}
h\left(e_{i}\right)=\left(a_{i, 1}+\left(t_{1}\right), \ldots, a_{i, m}+\left(t_{m}\right)\right), \\
e_{i}=\left(\left(k_{1}\right), \ldots,\left(k_{i-1}\right), 1+\left(k_{i}\right),\left(k_{i+1}\right), \ldots,\left(k_{n}\right)\right), \quad i=1, \ldots, n .
\end{gathered}
$$

Она обладает следующими свойствами:

$$
\frac{t_{j}}{\left(k_{i}, t_{j}\right)} \mid a_{i, j}
$$

для любых $i=1, \ldots, n, j=1, \ldots, m$ (здесь $\left(k_{i}, t_{j}\right)$ - наибольший общий делитель чисел $k_{i}$ и $t_{j}$, а $a \mid b$ обозначает, что $a$ делит $\left.b\right), h\left(\left(x_{1}+\left(k_{1}\right), \ldots, x_{n}+\left(k_{n}\right)\right)\right)=$ $=\left(x_{1}, \ldots, x_{n}\right) A_{h}$ для любых $x_{1}, \ldots, x_{n} \in \mathbf{Z}$. Следовательно, матрица $A_{h}$ однозначно определяет сам гомоморфизм $h$. Обратно, если $A=\left(a_{i, j}+\left(t_{j}\right)\right)_{n \times m}-$ произвольная матрица со свойством (12), то соответствие $h_{A}: X \rightarrow Y$, определяемое равенством

$$
h_{A}\left(\left(x_{1}+\left(k_{1}\right), \ldots, x_{n}+\left(k_{n}\right)\right)\right)=\left(x_{1}, \ldots, x_{n}\right) A,
$$

является гомоморфизмом групп. Таким образом, между $\operatorname{Hom}(X, Y)$ и всеми матрицами со свойством (12) существует взаимно-однозначное соответствие и, значит,

$$
\begin{aligned}
|\operatorname{Hom}(X, Y)|= & \prod\left(k_{i}, t_{j}\right), \\
& i=1, \ldots, n, \\
& j=1, \ldots, m,
\end{aligned}
$$

Следующая, доказанная в [5], теорема распространяет известный результат работы [1] для булевых функций на случай абелевых групп.

Теорема 2. Если $k_{i} \mid t_{j}$ для любых $i=1, \ldots, n, j=1, \ldots, m$, то для любой $f: X \rightarrow Y$ набор чисел

$$
(P(f=y+h) \mid y \in Y, h \in \operatorname{Hom}(X, Y))
$$

однозначно определяет функиию $f$.

Следующие две теоремы (см. [9], [5]) сводят случай многомерного $Y$ к одномерному и обобщают теорему 3 из [3].

Теорема 3. Если $k_{i}=t_{j}=t$ для любых $i=1, \ldots, n, j=1, \ldots, m$, то для любой $f: X \rightarrow Y$ следующие свойства равносильны:

(1) $f$ - сбалансированная;

(2) $h f-$ сбалансированная для любого эпиморфизма $h: Y \rightarrow Z /(t)$.

Теорема 4. Если $k_{i}=t_{j}=t$ для любых $i=1, \ldots, n, j=1, \ldots, m$, то для любой $f: X \rightarrow Y$ следующие свойства равносильны:

(1) $f \in B(X, Y)$;

(2) $h f \in B(X, \boldsymbol{Z} /(t))$ для любого эпиморфизма $h: Y \rightarrow \boldsymbol{Z} /(t)$. 
Лемма 1. Следуюшие свойства равносильны:

(a) для любых $x \in X \backslash\{0\}, y \in Y$ сущиествует $h \in \operatorname{Hom}(X, Y)$ такой, что $h(x)=y$

(б) $k_{1}=\ldots=k_{n}=t_{1}=\ldots t_{m}=p-$ простое число.

Доказательство. (б) $\Rightarrow$ (а) очевидно, так как в случае (б) гомоморфизмы являются линейными отображениями (функциями) векторных пространств над полем $\boldsymbol{Z} /(p)$.

Обратно, пусть выполнено (а). Тогда, в силу (12), $t_{j} \mid k_{i}$ для любых $i=1, \ldots, n, j=1, \ldots, m$. Для любого $h \in \operatorname{Hom}(X, Y)$ в $h\left(t_{j} e_{i}\right) j$-я координата равна 0 и, следовательно, $t_{j}=k_{i}=p=c_{1} c_{2}$, где $c_{1}>1$. Тогда $c_{1} h\left(c_{2} e_{1}\right)=0$ для любого $h \in \operatorname{Hom}(X, Y)$ и, следовательно, $c_{2}=1$. Лемма доказана.

Условие (a) существенно для дальнейших рассуждений. Поэтому далее рассмотрим только случай выполнения условия (б) леммы $1, p>1$, который назовем примарным (или $p$-примарным), при этом функции из $Y^{X}-$ примарными (или р-примарными). В этом случае гомоморфизмы из $\operatorname{Hom}(X, Y)$ являются линейными функциями (отображениями векторных пространств) и

$$
|\operatorname{Hom}(X, Y)|=|Y|^{n}=p^{n m} .
$$

2-примарный случай называют двоичным. Двоичный случай для $m=1$ называют булевым. С практической точки зрения оба эти случая наиболее интересны.

Лемма 2 (см. [5]). Для любой примарной $f: X \rightarrow Y$

$$
|Y|^{-n} \sum_{h \in \operatorname{Hom}(X, Y)} \delta(f, h)=|X|^{-1} .
$$

Доказательство этого равенства, приводимое ниже, проще соответствующего доказательства работы [5] и не использует коэффициенты Фурье.

Для любого $a \in X \backslash\{0\}$ отображение $\operatorname{Hom}(X, Y) \rightarrow Y$, где $h \mapsto h(a)$ является гомоморфизмом групп, который сюръективен в силу леммы 1 . Тогда, в силу (8),

$$
\begin{gathered}
|Y|^{-n} \sum_{h \in \operatorname{Hom}(X, Y)} \delta(f, h)= \\
=|Y|^{-n}|Y|(|X|(|Y|-1))^{-1}\left(|Y|^{n}\left(1-|Y|^{-1}\right)+\sum_{0 \neq a \in X} \sum_{h \in \operatorname{Hom}(X, Y)}\left(P\left(f a^{+}-f=h(a)\right)-|Y|^{-1}\right)\right)= \\
=|Y|^{-n}|Y|(|X|(|Y|-1))^{-1}\left(|Y|^{n}\left(1-|Y|^{-1}\right)+(|X|-1)\left(|Y|^{n-1}-|Y|^{n}|Y|^{-1}\right)\right)=|X|^{-1} .
\end{gathered}
$$

Лемма доказана.

Из этой леммы получаем оценку минимальной близости функций к линейным функциям. 
Теорема 5. Для примарного случая

$$
\delta_{0}(X, Y) \geq|X|^{-1} \text {. }
$$

Теорема 5 и формула (9) обосновывают теорему 6.

Tеорема 6. $B$ примарном случае, если $B(X, Y) \neq \varnothing$, то $B(X, Y) \subseteq M(X, Y)$ $u \delta_{0}(X, Y)=|X|^{-1}$.

В теореме 7 собраны критерии минимальности примарных функций для случая, когда $\delta_{0}(X, Y)$ минимальна (т. е. выполняется последнее равенство).

Теорема 7. Для любой р-примарной $f: X \rightarrow Y$ следующие утверждения равносильны:

(1) $\delta_{0}(X, Y)=|X|^{-1}$ и $f \in M(X, Y)$;

(2) $\delta(f, h)=|X|^{-1}$ для любого $h \in \operatorname{Hom}(X, Y)$;

(3) $\delta(f, h)=\delta\left(f, h^{\prime}\right)$ для любых $h, h^{\prime} \in \operatorname{Hom}(X, Y)$;

(4) $\sum_{0 \neq a \in X}\left(P\left(f a^{+}-f=h(a)\right)-|Y|^{-1}\right)=0$ для любого $h \in \operatorname{Hom}(X, Y)$;

(5) $\sum_{0 \neq a \in X}\left(P\left(f a^{+}-f=h(a)\right)-|Y|^{-1}\right)=\sum_{0 \neq a \in X}\left(P\left(f a^{+}-f=h^{\prime}(a)\right)-|Y|^{-1}\right)$ для любых $h, h^{\prime} \in \operatorname{Hom}(X, Y)$;

(6) $\sum_{0 \neq c \in Z /(p)}\left(P\left(f(c a)^{+}-f=c y\right)-|Y|^{-1}\right)=0$ для любых $a \in X \backslash\{0\}, y \in Y$;

(7) $\sum_{0 \neq c \in Z /(p)}\left(P\left(f(c a)^{+}-f=c y\right)-|Y|^{-1}\right)=\sum_{0 \neq c \in Z /(p)}\left(P\left(f(c a)^{+}-f=c y^{\prime}\right)-\right.$ $\left.-|Y|^{-1}\right)$ для любых $a \in X \backslash\{0\}, y, y^{\prime} \in Y$.

Доказательство. $(1) \Rightarrow(2)-$ следует из определения минимальности и леммы 2. (2) $\Rightarrow(3)-$ очевидно. $\quad(3) \Rightarrow(1)-$ следует из леммы 2 , теоремы 5 и определения минимальности. Из формулы (8) следуют $(2) \Leftrightarrow(4)$ и (3) $\Leftrightarrow(5)$. Импликации (6) $\Rightarrow(7)$ и (7) $\Rightarrow(5)$ очевидны.

Осталось доказать $(4) \Rightarrow(6)$. При $n=1$ импликация очевидна. Пусть $n \geq 2$, и пусть $a_{1}, \ldots, a_{n}$ - любой базис пространства $X$. Для любых $y_{1}, \ldots, y_{n} \in Y$ определим $h_{y_{1}, \ldots, y_{n}}: X \rightarrow Y$ равенством

$$
h_{y_{1}, \ldots, y_{n}}(a)=\sum_{i=1}^{n} c_{i} y_{i}
$$

для всех $a=\sum_{i=1}^{n} c_{i} a_{i}, \in X, \quad c_{1}, \ldots, c_{n} \in \mathbf{Z} /(p)$, так что $h_{y_{1}, \ldots, y_{n}} \in \operatorname{Hom}(X, Y)$. Тогда

$$
0=|Y|^{n-1} 0=\sum_{0 \neq a \in X} \sum_{y_{2}, \ldots, y_{n} \in Y}\left(P\left(f a^{+}-f=h_{y_{1}, \ldots, y_{n}}(a)\right)-|Y|^{-1}\right)=
$$




$$
\begin{gathered}
=\sum_{0 \neq c_{1} \in Z /(p)}|Y|^{n-1}\left(P\left(f\left(c_{1} a_{1}\right)^{+}-f=c_{1} y_{1}\right)-|Y|^{-1}\right)+ \\
\left.+\sum_{\overline{0} \neq\left(c_{2}, \ldots, c_{n}\right) \in(Z /(p))^{n-1}, c_{1} \in Z /(p)} \sum_{y_{2}, \ldots, y_{n} \in Y}\left(P\left(f a^{+}-f=\sum_{i=1}^{n} c_{i} y_{i}\right)\right)-|Y|^{-1}\right)= \\
=|Y|^{n-1} \sum_{0 \neq c_{1} \in Z /(p)}\left(P\left(f\left(c_{1} a_{1}\right)^{+}-f=c_{1} y_{1}\right)-|Y|^{-1}\right)+ \\
\left.+\sum_{\overline{0} \neq\left(c_{2}, \ldots, c_{n}\right) \in(Z /(p))^{n-1}, c_{1} \in Z /(p)} \sum_{y_{2}, \ldots, y_{n} \in Y}\left(P\left(f a^{+}-f=c_{1} y_{1}+\sum_{i=2}^{n} c_{i} y_{i}\right)\right)-|Y|^{-1}\right)= \\
=|Y|^{n-1} \sum_{0 \neq c_{1} \in Z /(p)}\left(P\left(f\left(c_{1} a_{1}\right)^{+}-f=c_{1} y_{1}\right)-|Y|^{-1}\right)+ \\
\sum_{\overline{0} \neq\left(c_{2}, \ldots, c_{n}\right) \in(Z /(p))^{n-1}, c_{1} \in Z /(p)} \quad|Y|^{n-2} \sum_{y \in Y}\left(P\left(f a^{+}-f=y\right)-|Y|^{-1}\right)= \\
=|Y|^{n-1} \sum_{0 \neq c_{1} \in Z /(p)}\left(P\left(f\left(c_{1} a_{1}\right)^{+}-f=c_{1} y_{1}\right)-|Y|^{-1}\right) .
\end{gathered}
$$

Теорема доказана.

Теорема 1 означает, что свойство абсолютной негомоморфности (т. е. бентовости) наследуется гомоморфными образами, а также то, что бентфункции не имеют нетривиальных гомоморфных образов с $\left|X^{\prime}\right|<|X|$. Покажем, что это справедливо и для свойства минимальности.

Tеорема 8. Если в примарном случае $\delta_{0}(X, Y)=|X|^{-1}, f \in M(X, Y), f^{\prime}: X^{\prime} \rightarrow Y^{\prime}-$ гомоморфный образ функиии $f u\left|Y^{\prime}\right|>1$, то

$$
\left|X^{\prime}\right|=|X|, \quad \delta_{0}\left(X^{\prime}, Y^{\prime}\right)=|X|^{-1} \text { u } f^{\prime} \in M\left(X^{\prime}, Y^{\prime}\right) .
$$

Доказательство. Для любых $a \in X \backslash\{0\}, y^{\prime} \in Y^{\prime}$ по формуле (11)

$$
\begin{gathered}
\sum_{0 \neq c \in Z /(p)}\left(P^{\prime}\left(f^{\prime}(c \alpha(a))+-f^{\prime}=c y^{\prime}\right)-\left|Y^{\prime}\right|^{-1}\right)= \\
=\sum_{0 \neq c \in Z /(p)} \sum_{y \in \beta^{-1}\left(y^{\prime}\right)}\left(P(f(c a)+-f=c y)-|Y|^{-1}\right)=0
\end{gathered}
$$

в силу утверждения (6) теоремы 7. Тогда $\alpha$ - изоморфизм, утверждение (6) теоремы 7 выполнено для $X^{\prime}, Y^{\prime}, f^{\prime}$ и теорема доказана.

Утверждение, эквивалентное теореме 8 для $Y^{\prime}=\boldsymbol{Z} /(p)$, в терминах коэффициентов Фурье доказано В. А. Шишкиным в 2008 году.

Следующая теорема обобщает хорошо известный для булева случая факт (см., например, [2]). 
Теорема 9. Для р-примарного случая, $p \in\{2,3\}$, следующие утверждения равносильны:

(1) $B(X, Y) \neq \varnothing$;

(2) $\delta_{0}(X, Y)=|X|^{-1}$;

(3) $B(X, Y)=M(X, Y)$.

Доказательство. (3) $\Rightarrow(1)-$ очевидно. (1) $\Rightarrow(2)-$ следует из теоремы 6. Докажем импликацию (2) $\Rightarrow(3)$. При $p=2$ она следует из утверждения (6) теоремы 7. Пусть $p=3$. Для любого $p$ выполняются равенства

$$
\begin{gathered}
P\left(f(-c a)^{+}-f=-c y\right)-|Y|^{-1}=P\left(f-f(c a)^{+}=-c y\right)-|Y|^{-1}= \\
=P\left(f(c a)^{+}-f=c y\right)-|Y|^{-1} .
\end{gathered}
$$

Пусть $f$ - минимальная. Тогда из утверждения (6) теоремы 7 получаем, что для любых $a \in X \backslash\{0\}, y \in Y$

$$
0=\sum_{c \in\{1,-1\}}\left(P\left(f(c a)^{+}-f=c y\right)-|Y|^{-1}\right)=2\left(P\left(f(a)^{+}-f=y\right)-|Y|^{-1}\right),
$$

и, следовательно, $f$ - абсолютно негомоморфная. Теорема доказана.

В [3] доказано, что если $p=2$ и $2 m \mid n$ или $p \geq 3$ и $m \mid n$, то $B(X, Y) \neq \varnothing$.

В заключение введем следующие обозначения и терминологию.

В случае выполнения условия

$$
\delta_{0}(X, Y)=|X|^{-1}
$$

минимальные функции назовем абсолютно минимальными (это определение сужает определение «абсолютной минимальности» в работе [5]). Множество всех абсолютно минимальных функций из $Y^{X}$ обозначим через $A(X, Y)$, так что

$$
\begin{aligned}
& M(X, Y)=A(X, Y), \text { если } \delta_{0}(X, Y)=|X|^{-1}, \\
& A(X, Y)=\varnothing, \quad \text { если } \delta_{0}(X, Y)>|X|^{-1} .
\end{aligned}
$$

Для любой $f: X \rightarrow Y$ обозначим

$$
\begin{gathered}
\bar{\delta}(f)=|X|^{-1} \sum_{a \in X} \delta\left(f a^{+}, f\right), \\
\delta_{1}(X, Y)=\min _{f \in Y^{X}} \bar{\delta}(f) .
\end{gathered}
$$


Максимально негомоморфными назовем функции $f \in Y^{X}$, для которых

$$
\bar{\delta}(f)=\delta_{1}(X, Y)
$$

Множество всех максимально негомоморфных функций из $Y^{X}$ обозначим через $N(X, Y)$, так что $N(X, Y) \neq \varnothing$ и

$$
\begin{aligned}
& N(X, Y)=B(X, Y), \text { если } \delta_{1}(X, Y)=|X|^{-1}, \\
& B(X, Y)=\varnothing, \quad \text { если } \delta_{1}(X, Y)>|X|^{-1} .
\end{aligned}
$$

Представляется справедливой следующая

Гипотеза. Для $p$-примарного случая и любого $p$

$$
\begin{aligned}
& A(X, Y)=B(X, Y), \\
& M(X, Y)=N(X, Y) .
\end{aligned}
$$

Теорема 9 равносильна выполнению равенства (14) для $p \in\{2,3\}$.

Наконец, заметим, что (в отличие от приведенного выше общепринятого определения бент-функции через равномодульность коэффициентов Фурье) в [6] для произвольного простого $p$ и в [7] для $p=2$ бент-функциями названы абсолютно минимальные функции. При этом в теореме 3.2 в [6] со ссылкой на [5] фактически утверждается, что утверждение теоремы 9 справедливо для любого простого $p$ (что автору не удалось доказать ни в [5], ни здесь). Последствием этого явилась необоснованность распространения некоторых свойств бент-функций на абсолютно минимальные функции. Теорема 9 настоящей работы обосновывает такое распространение на случаи $p=2,3$ в работах [6] и [7]. Отметим, что основные результаты [6] относятся к случаю $p=2$.

\section{Список литературы}

1. Golomb S. W. On the classification of Boolean functions. - IRE Trans. Circuit Theory, 1959, v. 1, no. 6, p. 10-27.

2. Rothaus O. S. On «bent» functions. - J. Comb. Theory, Ser. A, 1976, v. 20, no. 3, p. 300-305.

3. Амбросимов А. С. Свойства бент-функций $q$-значной логики над конечными полями. - Дискретная математика, 1994, т. 6, вып. 3, с. 50-60.

4. Логачёв О. А., Сальников А. А., Ященко В. В. Бент-функции на конечной абелевой группе. - Дискретная математика, 1997, т. 9, вып. 4, с. 3-20.

5. Солодовников В.И. Бент-функции из конечной абелевой группы в конечную абелеву группу. - Дискретная математика, 2002, т. 14, вып. 1, c. $99-113$. 
6. Кузьмин А. С., Нечаев А. А., Шишкин В. А. Бент- и гипербент-функции над конечным полем. - Труды по дискретной математике, 2007, т. 10. М.: ФИЗМАТЛИТ, с. 97-122.

7. Кузьмин А. С., Нечаев А. А., Шишкин В. А. Параметры (гипер-) бентфункций над полем из $2^{l}$ элементов. - Труды по дискретной математике, 2008, т. 11, вып. 1. - М.: ФИЗМАТЛИТ, с. 47-59.

8. Kumar P. V., Scholts R. A., Welch L. R. Generalized bent functions and their properties. - J. Comb. Theory, Ser. A, 1985, v. 40, no. 1, p. 90-107.

9. Nyberg $K$. Perfect nonlinear S-boxes. - Adv. in Cryptology - EUROCRYPT'91. Lect. Notes in Comput. Sci., 1991, v. 547, p. 378-386.

10. Токарева Н. Н. Нелинейные булевы функции: бент-функции и их обобщения. - Saarbrucken: LAP, 2011, 180 c. 